\title{
ONE MORE METHOD TO CONSTRUCT THE IRREDUCIBLE UNITARY REPRESENTATIONS OF NILPOTENT LIE GROUPS
}

\author{
A. A. ASTANEH \\ (Received 10 September 1984) \\ Communicated by W. Moran
}

\begin{abstract}
In this paper one more canonical method to construct the irreducible unitary representations of a connected, simply connected nilpotent Lie group is introduced. Although we use Kirillov's analysis to deduce this procedure, the method obtained differs from that of Kirillov's, in that one does not need to consider the coadjoint representation of the group in the dual of its Lie algebra (in fact, neither does one need to consider the Lie algebra of the group, provided one knows certain connected subgroups and their characters). The method also differs from that of Mackey's as one only needs to induce characters to obtain all irreducible representations of the group.
\end{abstract}

1980 Mathematics subject classification (Amer. Math. Soc.): 22 E 29.

\section{Introduction}

Throughout this paper $G$ will denote a connected, simply connected nilpotent Lie group and $\mathscr{G}$ its Lie algebra. It is well known that the irreducible unitary representations of $G$ can be obtained by Kirillov's orbital method (cf. [2]). One can also apply Mackey's theory to constrsuct the irreducible representations of $G$ (cf. [3]); since $G$ always contains a commutative connected (and hence closed) normal subgroup containing the nontrivial centre of $G$, and we know (cf. [1, Theorem 1]) that such a normal subgroup is always regularly embedded in $G$ in the sense of Mackey in [3]. The purpose of this paper is to introduce a third canonical way of constructing the irreducible representations of $G$. The method is deduced from the following three theorems.

C) 1986 Australian Mathematical Society $0263-6115 / 86 \$ A 2.00+0.00$ 


\section{The statements of the theorems}

The first theorem was proved by Dixmier in 1958 (cf. [1, Theorem 2]), using the earlier results of Mackey. His theorem is true in the more general case. We only need the following theorem.

THEOREM 1. Let $H$ be a commutative connected normal subgroup of $G$. Then every irreducible representation $U$ of $G$ is induced by a character $\chi$ of a connected subgroup $K$ of $G$ which contains $H$.

The second theorem we consider is the so called Shoda's criterion for irreducibility of monomial representations of $G$ which was proved by Pukanszky using Kirillov's results and was first published in his book (cf. [4, Theorem 2, Section 3, Chapter III, Part II]). The analogue of this theorem for finite groups was first stated and proved by Shoda around 1933.

THEOREM 2. Let $K$ be a connected subgroup of $G$, and $\chi$ a character of $K$. For $x \in G$ let $K_{x}=K \cap x^{-1} K x$ and denote by $\chi_{x}$ the character of $x^{-1} K x$ which is defined by

$$
\chi_{x}\left(k^{\prime}\right)=\chi\left(x k^{\prime} x^{-1}\right), \quad k^{\prime} \in x^{-1} K x .
$$

Then the representation $\operatorname{ind}_{K}^{G} \chi$ is irreducible if and only if for each $x \notin K$ the characters $\chi$ and $\chi_{x}$ have distinct restrictions to $K_{x}$.

We next extend another criterion of Shoda to connected, simply connected nilpotent Lie groups by using Kirillov's results.

THEOREM 3. Let $K_{i}, i=1,2$, be connected subgroups of the same dimension of $G$, and let $\chi^{i}$ be a character of $K_{i}$ such that ind ${ }_{K_{i}}^{G} \chi^{i}$ is irreducible. Then

$$
\operatorname{ind}_{K_{1}}^{G} \chi^{1} \cong \operatorname{ind}_{K_{2}}^{G} \chi^{2}
$$

if and only if there exists $x \in G$ such that the respective characters $\chi^{1}$ and $\chi_{x}^{2}$ of $K_{1}$ and $x^{-1} K_{2} x$ have the same restriction to $K_{1} \cap x^{-1} K_{2} x$, where $\chi_{x}^{2}$ is defined as (1) in Theorem 2.

Proof. For $i=1,2$, let $f_{i}$ be the functional on $\mathscr{G}$ from which $\chi^{i}$ is defined by (see [4, Section 3, Chapter III, Part II])

$$
\chi^{i}(\exp k)=e^{i f_{i}(k)}, \quad k \in \mathscr{K}_{i},
$$


where $\mathscr{X}_{i}$ is the Lie algebra of $K_{i}$. We also know (cf. [4, Theorem 1, Section 3, Chapter III, Part II]) that (2) holds if and only if there exists $x \in G$ such that

$$
f_{1}=\operatorname{Coad}(x) f_{2}
$$

where Coad denotes the coadjoint representation of $G$ in the vector space dual of $\mathscr{G}$. On the other hand (4) holds if and only if

$$
f_{1}(k)=f_{2}\left(\operatorname{Ad}\left(x^{-1}\right) k\right)
$$

for all $k \in \mathscr{K}_{1} \cap \operatorname{Ad}(x) \mathscr{K}_{2}$. Since the Lie algebra of $K_{1} \cap x K_{2} x^{-1}$ is identified with $\mathscr{K}_{1} \cap \operatorname{Ad}(x) \mathscr{K}_{2}$ (see [4, D, Section 1, Chapter I, Part II]), having also (3) in mind, we have that (5) holds if and only if

$$
\chi^{1}(\exp k)=\chi^{2}\left(x^{-1} \exp (k) x\right)
$$

for all $\exp (k) \in K_{1} \cap x K_{2} x^{-1}$. Since (6) means that $\chi^{1}$ and $\chi_{x}^{2}$ have the same restrictions to $K_{1} \cap x K_{2} x^{-1}$ the proof of the theorem is complete.

\section{The deduction of the procedure}

The above three theorems suggest the following procedure to obtain the irreducible unitary representations of $G$. Find a connected commutative normal subgroup of $G$, preferably of the largest dimension possible (as we mentioned in the introduction such an $H$ always exists). For each $n=\operatorname{dim} H,(\operatorname{dim} H)+$ $1, \ldots, \operatorname{dim} G$, let the set $\mathscr{S}_{n}$ denote the collection of all connected subgroups $K$ of $G$ which contain $H$ and have dimension $n$. Let also $\mathscr{C}_{n}$ denote the family of all characters of the subgroups belonging to $\mathscr{S}_{n}$ which satisfy the condition of Theorem 2. Theorems 1 and 2 ensure that if we induce all elements of all $\mathscr{C}_{n}$ 's to $G$, then (up to equivalence) we get all irreducible representations of $G$. To ensure that in the process of inducing these characters we do not get undue equivalent irreducible representations we first note (see, $[4$, the proof of Theorem 1 , Section 3, Chapter III, Part II]) that this may only happen between the characters belonging to the same $\mathscr{C}_{n}$. On the other hand the criterion of Theorem 3 clearly defines an equivalence relation in each $\mathscr{C}_{n}$. Let $\left\{\left[C_{n}\right]\right\}$ denote the set of all equivalence classes in $\mathscr{C}_{n}$ under this relation. Then Theorem 3 ensures that if we induce only one character from each member of $\left[C_{n}\right]$ we do not get undue equivalent representations.

\section{An example}

Let $G$ denote the nilpotent Lie group consisting of all real matrices

$$
\left(\begin{array}{llll}
1 & x & z & w \\
0 & 1 & y & v \\
0 & 0 & 1 & u \\
0 & 0 & 0 & 1
\end{array}\right)
$$


Let us write $[x, y, z, u, v, w]$ for the above element of $G$. Choose the subgroup of all elements of the form $[0, y, z, 0, v, w]$ as the subgroup $H$ of Theorem 1 . Then $\mathscr{S}_{4}$ consists only of $H$ itself. Let $\chi^{\nu, \lambda, \beta, \gamma}$ denote a typical character of $H$ which is defined by

$$
\chi^{\nu, \lambda, \beta, \gamma}([0, y, z, 0, v, w])=e^{i(v y+\lambda z+\beta v+\gamma w)} .
$$

Applying the criterion of Theorem 2 it is easy to see that

$$
\mathscr{C}_{4}=\left\{\chi^{\nu, \lambda, \beta, \gamma}: \gamma \neq 0\right\} .
$$

On the other hand, for arbitrary reals $\lambda, \beta$ and $\gamma \neq 0, \chi^{\nu, \lambda, \beta, \gamma}$ and $\chi^{\nu, 0,0, \gamma}$ belong to the same member of $\left[C_{4}\right]$; indeed $\chi_{[\beta, 0,0, \lambda, 0,0]}^{\nu, 0, \gamma}$ and $\chi^{\nu, \lambda, \beta, \gamma}$ coincide on $H$. Hence $\left\{\chi^{\nu, 0,0, \gamma}: \gamma \neq 0\right\}$ consists of one and only one character from each member of $\left[C_{4}\right]$. We then induce the elements of this set to get the following representations of $G$ in $L^{2}\left(\mathbb{R}^{2}\right)$,

$$
\left(\Pi^{\nu, \gamma}(g) f\right)(t, s)=e^{i(\nu y+\gamma[w+t v-(z+t y)(s+u)\}} f(t+x, s+u), \quad \nu \in \mathbb{R}, \gamma \neq 0,
$$

where $g=[x, y, z, u, v, w]$. Next we observe that $\mathscr{S}_{5}$ consists of the subgroups $K_{1}$, $K_{2}$ and also of all $K_{a}, a \neq 0$, with the respective typical elements [0,y,z,u,v,w], $[x, y, z, 0, v, w]$ and $[x, y, z, a x, v, w]$. Denote by $\chi^{\nu, \lambda, \alpha}, \phi^{\mu, \nu, \beta}$ and $\psi^{\mu, \nu, \beta}$ the typical characters of $K_{1}, K_{2}$ and $K_{a}$, respectively, which are defined by

$$
\begin{aligned}
& \chi^{\nu, \lambda, \alpha}([0, y, z, u, v, w])=e^{i(\nu y+\lambda z+\alpha u)}, \\
& \phi^{\mu, \nu, \beta}([x, y, z, 0, v, w])=e^{i(\mu x+\nu y+\beta v)},
\end{aligned}
$$

and

$$
\psi_{a}^{\mu, \nu, \beta}([x, y, z, a x, v, w])=e^{i[\mu x+\nu y+a \beta(z-x y)+\beta v]} .
$$

Applying Theorem 2 we have that

$$
\mathscr{C}_{5}=\left\{\chi^{\nu, \lambda, \alpha}, \phi^{\mu, \nu, \beta}, \psi_{a}^{\mu, v, \beta}: a \lambda \beta \neq 0\right\} .
$$

It is also easy to check that for arbitrary $\nu$ and $a, \lambda, \beta \neq 0, \chi_{[\nu, 0,0,0,0,0]}^{0, \lambda}=\chi^{\nu, \lambda, \alpha}$, $\phi_{[0,0,0,-\nu, 0,0]}^{\mu, 0, \beta}=\phi^{\mu, \nu, \beta}$ and $\left(\psi_{a}^{\mu, 0, \beta}\right)_{[0,0,0,-\nu / \beta, 0,0]}=\psi_{a}^{\mu, \nu, \beta}$, so that it is enough to find the equivalence classes of the following subset of $\mathscr{C}_{5}$ :

$$
\mathscr{C}_{s}^{*}=\left\{\chi^{0, \lambda, \alpha}, \phi^{\mu, 0, \beta}, \psi_{a}^{\mu, 0, \beta}: a \lambda \beta \neq 0\right\} .
$$

Since $K_{1}, K_{2}$ and also $K_{a}$ are normal subgroups and since

$$
K_{1} \cap K_{2}=K_{1} \cap K_{a}=K_{2} \cap K_{a}=H,
$$

one immediately checks, by applying the criterion of Theorem 3 , that $\mathscr{C}_{5}{ }^{*}$ indeed contains one and only one character from each member of $\left\{\left[C_{5}\right]\right\}$. We therefore induce the characters $\chi^{0, \lambda, \alpha}, \phi^{\mu, 0, \beta}$ and $\psi_{a}^{\mu, 0, \beta}$ of $K_{1}, K_{2}$ and $K_{a}$ (in respective 
order) to obtain the following irreducible representations of $G$ in $L^{2}(\mathbb{R})$ : for $g=[x, y, z, u, v, w]$

$$
\left(U^{\lambda, \alpha}(g) f\right)(t)=e^{i \lambda[(z+t y)+\alpha u]} f(t+x), \quad \lambda \neq 0, \alpha \in \mathbb{R},
$$

$$
\left(W^{\mu, a \beta, \beta}(g) f\right)(t)=e^{i[\mu x+a \beta z+\beta(v-y u-t y)]} f(t+u-a x), \quad \alpha \beta \neq 0, \mu \in \mathbb{R} .
$$

Finally $\mathscr{S}_{6}$ consists of $G$ only, and the characters of $G$ are defined by

$$
\chi^{\mu, \nu, \alpha}([x, y, z, u, v, w])=e^{i(\mu x+\nu y+\alpha u)}, \quad \mu, \nu, \alpha \in \mathbb{R} .
$$

We end this section by mentioning that the representations (ii), (iii) and (iv) can be put into one formula; indeed for a real number $r \neq 0$, let $T^{r}$ be the unitary operator in $L^{2}(\mathbb{R})$ defined by

$$
\left(T^{r} f\right)(t)=(|r| / 2 \pi)^{1 / 2} \int_{\mathbb{R}} e^{i r t \tau} f(\tau) d \tau
$$

Then it is easy to see that the respective equivalent representations $U_{1}^{\lambda, \alpha}=$ $T^{\lambda} U^{\lambda, \alpha}\left(T^{\lambda}\right)^{-1}, V_{1}^{\mu, \beta}=\left(T^{\beta}\right)^{-1} V^{\mu, \beta} T^{\beta}$ and $W_{1}^{\mu, a \beta, \beta}=\left(T^{\beta}\right)^{-1} W^{\mu, a \beta, \beta} T^{\beta}$ to $U^{\lambda, \alpha}$, $V^{\mu, \beta}$ and $W^{\mu, a \beta, \beta}$ are defined by

$$
\left(U_{1}^{\lambda, \alpha}(g) f\right)(t)=e^{i \lambda(z-x y-t x)+\alpha \mu]} f(t+y), \quad \lambda \neq 0, \alpha \in \mathbb{R},
$$

(iii) ${ }^{*}$

$$
\left(V_{1}^{\mu, \beta}(g) f\right)(t)=e^{i[\mu x+\beta(v+t u)]} f(t+y), \quad \beta \neq 0, \mu \in \mathbb{R},
$$

$$
\left(W_{1}^{\mu, a \beta, \beta}(g) f\right)(t)=e^{i[\mu x+a \beta(z-x y-t x)+\beta(v+t u)]} f(t+y), \quad a \beta \neq 0, \mu \in \mathbb{R} .
$$

Now if we put $\lambda=a \beta$ in (iv)* then (ii)*-(iv)* can be considered as

(ii) $)^{* *} \quad\left(\rho^{\mu, \lambda, \alpha, \beta}(g) f\right)(t)=e^{i[\mu x+\lambda(z-x y-t x)+\beta(v+t u)+\alpha u]} f(t+y)$.

with the conditions $\lambda^{2}+\beta^{2} \neq 0, \alpha \beta=0$, and that $\mu=0$ whenever $\lambda \neq 0$ but $\beta=0$.

We also mention that the representations $\Pi^{\nu, \gamma}, \gamma \neq 0, \nu \in \mathbb{R}$, of $G$ given by (i) are equivalent to $\sigma^{\nu, \gamma}$ defined by

$$
\left(\sigma^{\nu, \gamma}(g) f\right)(t, s)=e^{i[\nu y+\gamma(w+t v+s u)]} f(t+x, s+z+t y),
$$

since $\sigma^{\nu, \gamma}=\left(T^{\gamma}\right)^{-1} \Pi^{\nu, \gamma} T^{\gamma}$ where

$$
\left(T^{\gamma} f\right)(t, s)=(|\gamma| / 2 \pi)^{1 / 2} \int_{\mathbf{R}} e^{i \gamma s \tau} f(t, \tau) d \tau
$$




\section{Acknowledgement}

I wish to thank Dr. S. Sankaran, under whose supervision this work was done as a part of my Ph.D. thesis at the University of London.

\section{References}

[1] J. Dixmier, 'Sur les représentations des groupes de Lie nilpotente I', Amer. J. Math. 81 (1959), $160-170$.

[2] A. A. Kirillov, 'Unitary representations of nilpotent Lie groups', Russian Math. Surveys 17 (1962), 53-104.

[3] G. W. Mackey, 'Unitary representations of group extensions I', Acta Math. 99 (1958), 265-311.

[4] L. Pukanszky, Leçons sur les représentations des groupes (Dunod, Paris, 1967).

Department of Mathematics

University of Mashhad

Mashhad

Iran 